\title{
Monotonic laterally loaded pile testing in a dense marine sand at Dunkirk
}

\author{
ROSS A. MCADAM*, BYRON W. BYRNE*, GUY T. HOULSBY*, WILLIAM J. A. P. BEUCKELAERS $\dagger$, \\ HARVEY J. BURD*, KENNETH G. GAVIN\$, DAVID J. P. IGOE§, RICHARD J. JARDINE\|, \\ CHRISTOPHER M. MARTIN*, ALASTAIR MUIR WOOD \\ JESPER SKOV GRETLUND**, DAVID M. G. TABORDA $\|$ and LIDIJA ZDRAVKOVIĆ $\|$
}

\begin{abstract}
The results obtained from a field testing campaign on laterally loaded monopiles, conducted at a dense sand site in Dunkirk, northern France are described. These tests formed part of the PISA project on the development of improved design methods for monopile foundations for offshore wind turbines. Results obtained from monotonic loading tests on piles of three different diameters $(0.273 \mathrm{~m}, 0.762 \mathrm{~m}$ and $2.0 \mathrm{~m})$ are presented. The piles had length-to-diameter ratios $(L / D)$ of between 3 and 10 . The tests consisted principally of the application of monotonic loads, incorporating periods of held constant load to investigate creep effects. The influence of loading rate was also investigated. Data are presented on the overall load-displacement behaviour of each of the test piles. Measured data on bending moments and inclinations induced in the piles are also provided. Inferences are made for the displacements in the embedded length of the piles. These field data will support the development of a new one-dimensional modelling approach for the design of monopile foundations for offshore wind turbines. They also form a unique database of field measurements in a dense sand, from lateral loading of piles at a vertical distance above the ground surface.
\end{abstract}

KEYWORDS: full-scale tests; model tests; offshore engineering; piles \& piling; soil/structure interaction

\section{INTRODUCTION}

A study (referred to as PISA) has been completed, employing field testing and three-dimensional (3D) finite-element modelling to develop a new approach for the design of monopile foundations for wind turbine support structures in shallow North Sea waters. An overview of the project, a general description of the field tests and details of site characterisation are given in the papers by Byrne et al. (2017) and Zdravković et al. (2019).

This paper provides a detailed description of the results of field tests on reduced-scale monopile foundations, loaded laterally, conducted at Dunkirk, in northern France, as part of the PISA study. A separate paper, by Byrne et al. (2019), describes a complementary set of tests conducted in a stiff, overconsolidated clay at Cowden, UK. The soil at the Dunkirk site consists principally of a dense Flandrian sand with a surface layer (about $3 \mathrm{~m}$ thick) of dense hydraulically placed sand with the same geological origin as the deeper Flandrian deposit.

Manuscript received 5 March 2018; revised manuscript accepted 11 July 2019. Published online ahead of print 15 November 2019.

Discussion on this paper closes on 1 March 2021, for further details see $\mathrm{p}$. ii.

Published with permission by the ICE under the CC-BY 4.0 license. (http://creativecommons.org/licenses/by/4.0/)

* Department of Engineering Science, University of Oxford, Oxford, UK.

$\dagger$ Jan De Nul Group, Aalst, Belgium; formerly Department of Engineering Science, Oxford University, Oxford, UK.

† Delft University of Technology, Delft, the Netherlands; formerly School of Civil Engineering, University College Dublin, Dublin, Ireland.

$\S$ Trinity College, Dublin, Ireland; formerly School of Civil Engineering, University College Dublin, Dublin, Ireland.

|| Department of Civil and Environmental Engineering, Imperial College London, London, UK.

I Wood Thilsted Partners, London, UK; formerly Ørsted Wind

Power, London, UK.

** Ørsted Wind Power, Copenhagen, Denmark.
Testing was conducted on piles with three different diameters $(0.273 \mathrm{~m}, 0.762 \mathrm{~m}$ and $2.0 \mathrm{~m})$ using the test protocols and instrumentation described in the paper by Burd et al. (2019). Most of the tests were conducted at the same (controlled) value of ground-level pile velocity; a few tests were conducted at higher displacement rates to observe the effects of rate on the pile response. Repeatability tests were also conducted.

The Dunkirk field test data have been used to support the development of a new design approach (termed the 'PISA design model') for offshore monopile design. The development of this design model is beyond the scope of the current paper (although the general principles that have been employed in the development of the design model are outlined in the papers by Byrne et al. (2017) and Zdravković et al. (2019)). A description of the 3D finite-element model that has been employed to assist in the interpretation of the field data is given in the paper by Taborda et al. (2019).

\section{SPECIFICATION OF THE TEST PROGRAMME \\ Dunkirk test site}

Details of the procedures adopted to characterise the soil conditions at the Dunkirk site are provided in the papers by Zdravković et al. (2019) and Taborda et al. (2019). A summary of these site characterisation procedures is given below.

The Dunkirk test site is part of the extended beach area, situated on land owned by the Port Authority of Dunkirk. The soil is characterised as a dense marine Pleistocene deposit, predominantly consisting of Flandrian sand with a relative density assessed to be $D_{\mathrm{R}}=75 \%$. The top $3 \mathrm{~m}$ of the deposit consists of a dense hydraulic fill (assessed to have relative density $D_{\mathrm{R}}=100 \%$ ) placed several decades ago to raise the ground level.

The water table at the time of the test programme was at approximately $5.4 \mathrm{~m}$ depth. This implies the existence of a potentially unsaturated region of sand in the top few metres of soil. The conditions within this surface layer were found to have a significant influence on the near-surface behaviour of 
the soil. During the test programme, for example, a gap was invariably observed to form on the active pile face during loading and on the passive pile face during unloading. The gaps would typically collapse several days after completion of the tests (potentially due to dissipation of the pore pressure suctions). This region required careful consideration in developing the 3D finite-element models (Taborda et al., 2019) that were employed to support the interpretation of the field tests. Furthermore, since unsaturated surface layers are unlikely to be present at offshore sites, it is not straightforward to extrapolate the results of the field tests, directly, to the behaviour of full-scale monopiles. The PISA project employed an alternative framework, depicted in Fig. 3 in the paper by Zdravković et al. (2019), in which the field data are used to validate a finite-element model developed specifically for the conditions at the test sites. Once validated, the finite-element model is employed to predict the performance of full-scale monopiles.

Assessments of the small-strain shear modulus, $G_{0}$, at the Dunkirk site were supported by in situ seismic cone penetration tests (SCPTs) conducted specifically for the PISA research, in addition to Chow (1997) seismic cone tests. A set of isotropically consolidated, drained, triaxial

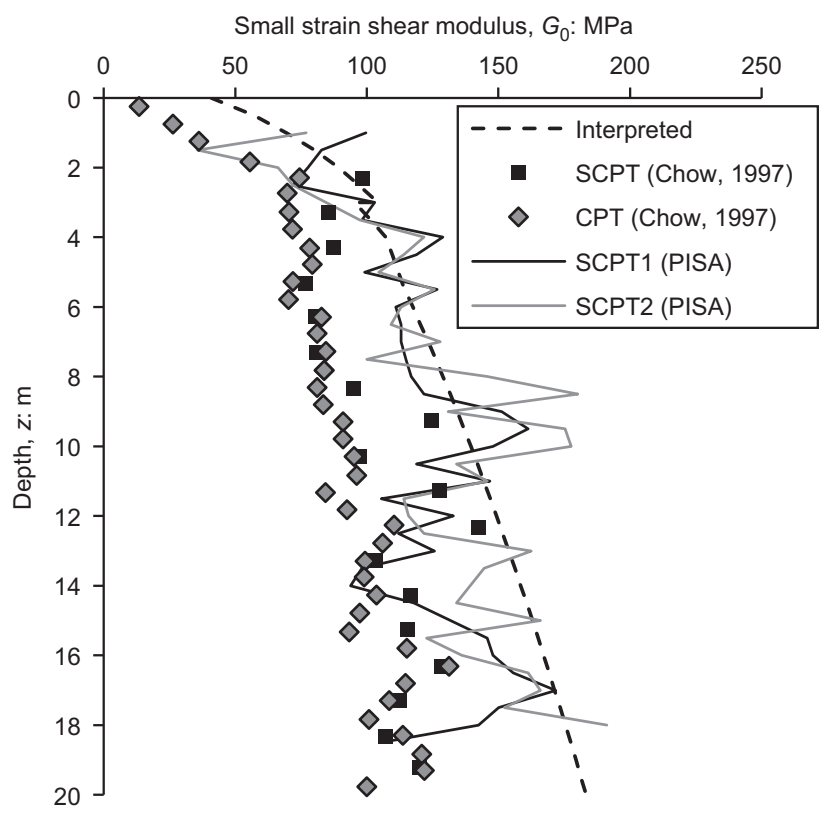

Fig. 1. Interpreted profile of small-strain shear modulus for the Dunkirk site. Also shown are shear modulus data determined from the seismic cone tests conducted by Chow (1997) tests conducted for the PISA project, employing local instrumentation, supported assessments of shear strength and volumetric behaviour of Dunkirk sand. Data from these tests were supplemented with $K_{0}$-consolidated triaxial tests on Dunkirk sand, collected by Aghakouchak (2015) during a project unrelated to PISA.

The characterisation of the depth variation of $G_{0}$ for the purposes of the current tests employed the Hardin (1978) correlation

$$
G_{0}=\frac{B p_{\text {ref }}}{0 \cdot 3+0 \cdot 7 e^{2}}\left(\frac{p^{\prime}}{p_{\text {ref }}}\right)^{0.5}
$$

where $p_{\text {ref }}$ is a reference pressure (taken to be $101.3 \mathrm{kPa}$ ); $p^{\prime}$ is the local value of mean effective stress; and $e$ is the void ratio. This correlation requires a single parameter, $B$, to be determined. This parameter cannot be determined straightforwardly in this case, since the various data sets imply slightly different values. As described in the paper by Taborda et al. (2019), however, $B=875$ was adopted as an appropriate value. The saturated bulk unit weight of the sand is $\gamma_{\text {sat }}=$ $17 \cdot 1 \mathrm{kN} / \mathrm{m}^{3}$ above the water table and $19.9 \mathrm{kN} / \mathrm{m}^{3}$ below the water table (Zdravković et al., 2019) and $K_{0}=0.4$ is assumed (Chow, 1997). The void ratio in the upper, $100 \%$ dense layer is $e=0.570$; the void ratio in the lower $75 \%$ dense material is $e=0.628$ (Taborda et al., 2019). To estimate the mean effective stress in the superficial (unsaturated) region, negative pore pressures (suctions) were assumed, as explained in the paper by Zdravković et al. (2019). Pore pressures were assumed to be hydrostatic below the water table. The interpreted $G_{0}$ profile determined on this basis is plotted in Fig. 1. Also shown in Fig. 1 are sets of data on $G_{0}$ inferred from new and Chow (1997) SCPT tests conducted at the Dunkirk site.

The triaxial tests conducted for the purposes of the PISA research were taken to sufficiently high strains to allow critical states to be identified. On this basis, the critical state friction angles in compression and extension were identified to be $32^{\circ}$ and $33^{\circ}$, respectively (Taborda et al., 2019).

Specification of test piles

The test piles employed for the tests were specified to be similar to those adopted for the field tests at Cowden. This meant that much of the instrumentation and loading equipment developed for the project could be deployed at both sites. The rationale for the choice of the pile configurations adopted for the tests is also discussed in the paper by Byrne et al. (2019).

The test piles are specified in Table 1, which lists both the nominal and installed values of embedded $L / D$. Nominal

Table 1. Test pile geometries and loading

\begin{tabular}{l|l|c|c|c|c|c|c|c|c|c}
\hline Pile & $D: \mathrm{m}$ & $\begin{array}{c}\text { Nominal } \\
L / D\end{array}$ & $\begin{array}{c}\text { Installed } L: \\
\mathrm{m}\end{array}$ & $\begin{array}{c}\text { Installed } \\
L / D\end{array}$ & $\begin{array}{c}t: \\
\mathrm{mm}\end{array}$ & $h: \mathrm{m}$ & $h / D$ & $\begin{array}{c}\text { Embedded } \\
\text { instruments }\end{array}$ & $\begin{array}{c}\text { Date } \\
\text { installed }\end{array}$ & $\begin{array}{l}\text { Date } \\
\text { tested }\end{array}$ \\
\hline DS1 & $0 \cdot 273$ & $5 \cdot 25$ & $1 \cdot 43$ & $5 \cdot 24$ & 7 & $5 \cdot 0$ & $18 \cdot 3$ & & & $08 / 11 / 14$ \\
DS2 & $0 \cdot 273$ & $5 \cdot 25$ & $1 \cdot 43$ & $5 \cdot 24$ & 7 & $5 \cdot 0$ & $18 \cdot 3$ & & $22 / 04 / 15$ \\
DS3 & $0 \cdot 273$ & 8 & $2 \cdot 18$ & 8 & 7 & $5 \cdot 0$ & $18 \cdot 3$ & & $08 / 11 / 14$ & $25 / 04 / 15$ \\
DS4 & $0 \cdot 273$ & 10 & $2 \cdot 73$ & 10 & 7 & $5 \cdot 0$ & $18 \cdot 3$ & & $08 / 11 / 14$ & $23 / 04 / 15$ \\
DM5 & $0 \cdot 762$ & 3 & $2 \cdot 27$ & $2 \cdot 98$ & 10 & $9 \cdot 99$ & $13 \cdot 1$ & & $08 / 11 / 14$ & $24 / 04 / 15$ \\
DM7 & $0 \cdot 762$ & 3 & $2 \cdot 24$ & $2 \cdot 94$ & 10 & $10 \cdot 00$ & $13 \cdot 1$ & $\mathrm{Y}$ & $10 / 11 / 14$ & $08 / 05 / 15$ \\
DM4 & $0 \cdot 762$ & $5 \cdot 25$ & $3 \cdot 98$ & $5 \cdot 22$ & 14 & $9 \cdot 98$ & $13 \cdot 1$ & $\mathrm{Y}$ & $10 / 11 / 14$ & $14 / 05 / 15$ \\
DM9 & $0 \cdot 762$ & $5 \cdot 25$ & $3 \cdot 96$ & $5 \cdot 20$ & 14 & $10 \cdot 00$ & $13 \cdot 1$ & & $10 / 1 / 14$ & $12 / 05 / 15$ \\
DM6 & $0 \cdot 762$ & $5 \cdot 25$ & $3 \cdot 99$ & $5 \cdot 24$ & 19 & $10 \cdot 00$ & $13 \cdot 1$ & $\mathrm{Y}$ & $10 / 11 / 14$ & $30 / 04 / 15$ \\
DM3 & $0 \cdot 762$ & 8 & $6 \cdot 02$ & $7 \cdot 90$ & 25 & $10 \cdot 06$ & $13 \cdot 2$ & $\mathrm{Y}$ & $06 / 11 / 14$ & $05 / 06 / 15$ \\
DL1 & 2 & $5 \cdot 25$ & $10 \cdot 61$ & $5 \cdot 30$ & 38 & $9 \cdot 90$ & $4 \cdot 95$ & $\mathrm{Y}$ & $14 / 11 / 14$ & $203 / 06 / 15$ \\
DL2 & 2 & $5 \cdot 25$ & $10 \cdot 57$ & $5 \cdot 29$ & 38 & $9 \cdot 89$ & $4 \cdot 95$ & $\mathrm{Y}$ & $13 / 11 / 14$ & $20 / 06 / 15$ \\
\hline
\end{tabular}

Notes: D, Dunkirk; S, small diameter $(0.273 \mathrm{~m})$; M, medium diameter $(0.762 \mathrm{~m})$; L, large diameter $(2.0 \mathrm{~m})$; 'Nominal $(L / D)$ ' refers to the initial specifications of the test piles; 'Installed $L$ ' and 'Installed $L / D$ ' refer to measured pile embedded lengths after installation. 
$L / D$ values are referenced in this paper for ease of comparison between the tests. The pile diameters, designated as small $(D=0.273 \mathrm{~m})$, medium $(D=0.762 \mathrm{~m})$ and large $(D=2.0 \mathrm{~m})$ are the same as those employed for the Cowden tests. The nominal values of embedded length-to-diameter ratio $(L / D)$ specified for the test piles $(3,5 \cdot 25$ and 8$)$ are also consistent with the Cowden tests, with the exception of pile DM3. This pile has a nominal $L / D$ of 8 , whereas the equivalent Cowden pile (CM3) has a nominal $L / D$ of 10 . Consistent with the approach adopted to specify the Cowden test piles, individual pile wall thicknesses for the current tests were selected on the basis of $3 \mathrm{D}$ finite-element predictions of the performance of each of the test piles (Zdravkovic et al., 2015). It was important for subsequent data processing that the pile wall thicknesses were sufficiently large to ensure that yielding of the pile wall did not occur in any of the tests. To maximise the resolution of the bending moments determined from strain gauges attached to the pile, however, a relatively small value of pile wall thickness is desirable. Values of pile wall thickness determined on the basis of these two considerations are specified in Table 1. The piles are thicker than the corresponding Cowden test piles for the large-diameter piles, and also the medium-diameter piles with relatively large values of $L / D$, due to the greater anticipated soil reactions in the Dunkirk tests.

The pile test configurations employed in the study were chosen to provide representative scaling of key geometric aspects of the problem. The height, $h$, above ground at which the lateral loading is applied in the tests was selected to provide values of normalised load eccentricity $h / D$ that are consistent with loading (due to wind and wave) applied to full-scale wind turbine support structures. The tests employed piles with length-to-diameter ratios $(L / D)$ in the range from 3 to 8 ; current monopile designs fall within this length-to-diameter ratio range (although increasingly towards the lower end). No attempt has been made to provide a complete scaling of the problem - for example in terms of the ratio of the bending stiffness of the pile to the stiffness of the soil. Instead, the pile tests are used to infer the behaviour of full-scale piles by way of 3D finite-element modelling (as outlined above).

\section{Installation procedures}

The medium- and large-diameter piles were installed in a two-stage process, with an initial vibration stage used to embed the piles to a stable depth $(1.0 \mathrm{~m}$ to $1.5 \mathrm{~m})$, followed by pile driving with a hydraulic hammer until reaching the target embedment. Impact driving was carried out using a Dawson HPH6500e piling hammer, with a conical follower for driving the large-diameter piles. It was assumed that the initial vibration stage has little influence on the pile-soil interaction and that the piles can be considered as being installed by way of typical impact methods, as employed offshore. In contrast, the small-diameter piles were vibrated to the target embedment.

\section{Monotonic tests}

A horizontal load $H$ was applied to the test piles from a reaction pile, at a height $h$ above ground, as shown in Fig. 2 (see also the paper by Burd et al. (2019)). Further details on the experimental apparatus and calculation of the ground-level pile response from the above-ground displacement transducer and inclinometer data are provided in the paper by Burd et al. (2019). The principal tests employed the maintained load monotonic loading scheme specified in Table 2 and illustrated in Fig. 3. Initial (load stage 0) and intermediate unload-reload loops were used to check the

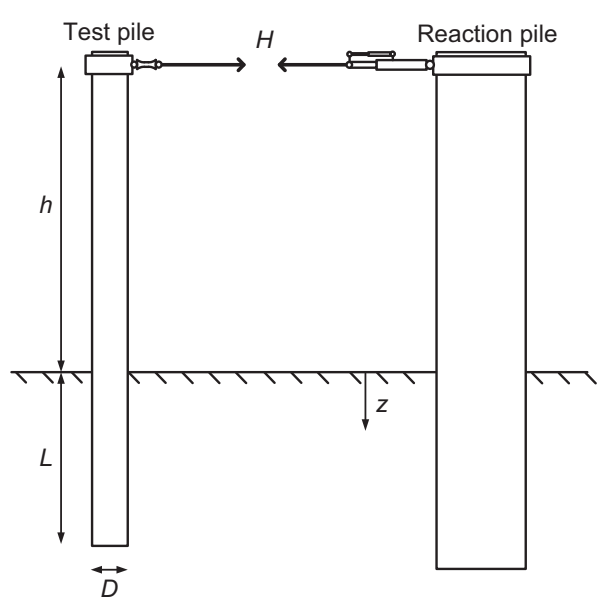

Fig. 2. Outline of the pile testing set-up. Further details of the loading configurations are given in the paper by Burd et al. (2019)

Table 2. Monotonic test load stages

\begin{tabular}{l|c|c}
\hline Stage number & $\begin{array}{c}\text { Loading rate: } \\
\mathrm{m} / \mathrm{min}\end{array}$ & $\begin{array}{c}\text { Normalised displacement, } \\
v_{\mathrm{G}} / D: \%\end{array}$ \\
\hline 0 & $D / 1000$ & $0 \cdot 1$ \\
1 & $D / 300$ & $0 \cdot 125$ \\
2 & $D / 300$ & $0 \cdot 5$ \\
3 & $D / 300$ & $1 \cdot 5$ \\
Unload-reload & $D / 500$ & $-\overline{ }$ \\
4 & $D / 300$ & $2 \cdot 5$ \\
5 & $D / 300$ & 5 \\
6 & $D / 300$ & $6 \cdot 5$ \\
7 & $D / 300$ & $8 \cdot 25$ \\
8 & $D / 300$ & 10 \\
9 & $D / 300$ & \\
Unload & $D / 500$ & \\
\hline
\end{tabular}

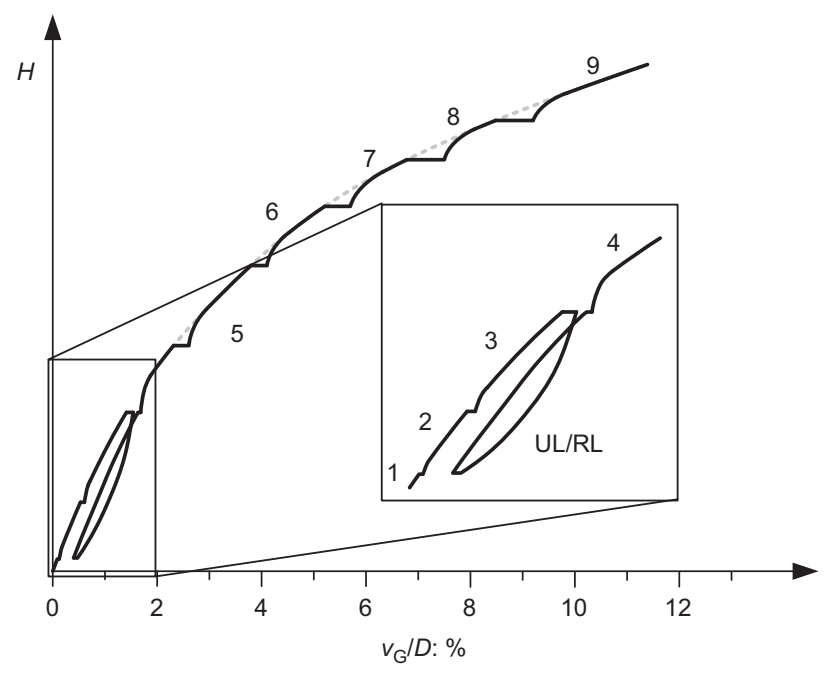

Fig. 3. Monotonic loading regime (UL, unload; RL, reload)

operation of the instrumentation and to observe the change in stiffness following the application of a small load, respectively. The Dunkirk tests were conducted after most of the testing at Cowden (Byrne et al., 2019) had been completed. Since much of the control system and data logging hardware used at Dunkirk had previously been employed at Cowden, the testing campaign at Dunkirk was 
able to benefit from experience, gained at Cowden, on procedures to tune the control system and to log the data.

A test was classified as complete when both $(a)$ the ground-level pile displacement had exceeded $v_{\mathrm{G}}=D / 10$ and (b) the ground-level pile rotation had exceeded $\theta_{\mathrm{G}}=2^{\circ}$.

\section{SMALL-DIAMETER $(D=0 \cdot 273 \mathrm{~m})$ TEST RESULTS}

In a similar process to the testing at Cowden (Byrne et al., 2019), piles were tested in order of increasing diameter to confirm the operation of the control system at each scale. The small-diameter piles $(D=0 \cdot 273 \mathrm{~m})$ were therefore the first to be tested. Piles with three different length-to-diameter ratios, $L / D=5 \cdot 25,8$ and 10 , were installed and tested. Two equivalent piles with a lengthto-diameter ratio of $5 \cdot 25$ (DS1 and DS2) were included in the test programme; these piles were tested at different velocities to explore the influence of rate effects on the pile response, as rate effects had been identified as significant in the previous tests in clay at Cowden, see the paper by Byrne et al. (2019).

The ground-level load-displacement response of the three monotonic small-diameter pile tests, excluding DS2 which was tested at an enhanced rate, are shown in Fig. 4. Fig. 4(a) shows the response of DS1 $(L / D=5 \cdot 25)$. It is clear that, in this case, significant lateral deformations occur during the creep stages, particularly at higher loads, where the overall pile response appears to soften. Fig. 4(b) shows the response of DS3 $(L / D=8)$. The stiffness of this pile was higher than

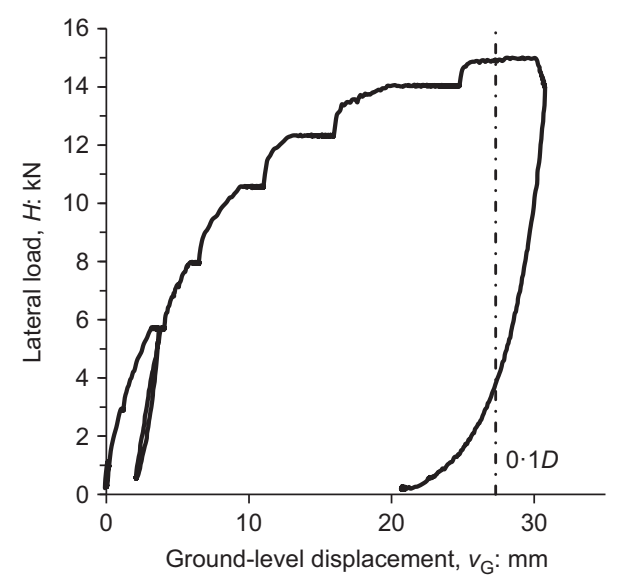

(a)

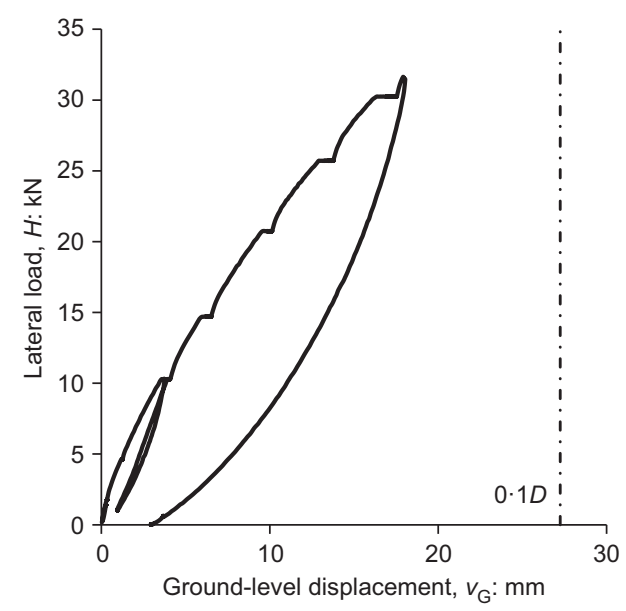

(c) had been anticipated when the tests were designed and, as a consequence, it seemed likely that initial yielding of the pile itself would occur before the usual ground-level displacement and rotation limits $\left(0 \cdot 1 D\right.$ and $2^{\circ}$, respectively) were reached. It was therefore decided to unload the pile prior to yielding of the pile steel (which was estimated to occur at a lateral load of $27 \mathrm{kN}$ ), to ensure that elastic behaviour of the pile could be assumed in the data-processing procedures. A similar approach was taken for DS4 $(L / D=10)$ to avoid the possibility of pile yielding.

Figure 4(d) shows a comparison between the three comparable small-diameter tests (with different length-to-diameter ratios). The similarity in the responses of the two longer piles suggests that for $L / D=8$ the pile is effectively fixed at depth; increasing the length to $L / D=10$, therefore, has minimal effect on the pile's performance. The softer response for $L / D=5 \cdot 25$ indicates that, in this case, base fixity does not apply. Moreover, the relatively soft response of the DS1 $(L / D=5 \cdot 25)$ pile at $v_{\mathrm{G}}=D / 10$ suggests that soil failure had occurred over a significant proportion of the embedded pile length.

Figure 5 shows the results from the small-diameter pile tests DS1, DS3 and DS4 during the initial load stages and unload-reload loop. The three responses are compared in Fig. 5(d); this figure indicates that the slope of the unload-reload loops for the piles are similar, although the shorter pile $(L / D=5 \cdot 25)$ indicates a backbone curve that is significantly softer than is observed in the other two tests.

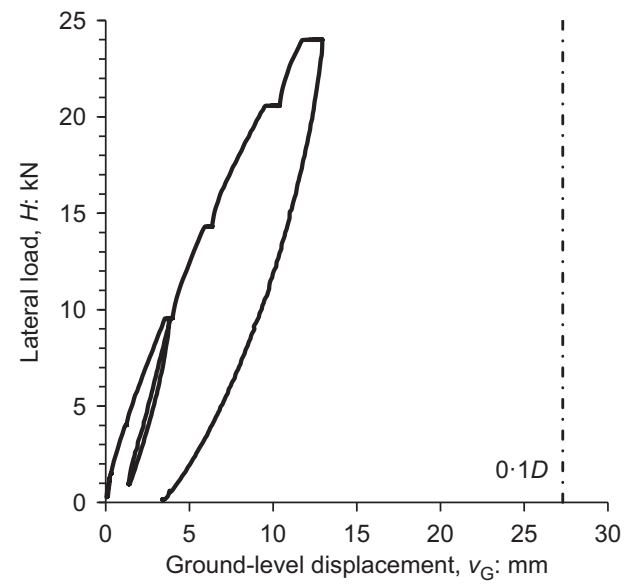

(b)

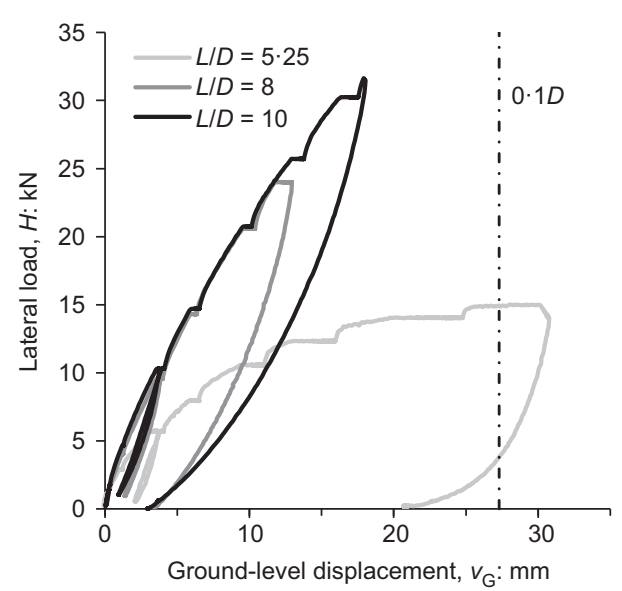

(d)

Fig. 4. Comparison of load-displacement response for $D=0 \cdot 273 \mathrm{~m}$ diameter piles with different length-to-diameter ratios: (a) $L / D=5 \cdot 25$ (DS1); (b) $L I D=8$ (DS3); (c) $L I D=10$ (DS4); (d) three $L I D$ ratios 


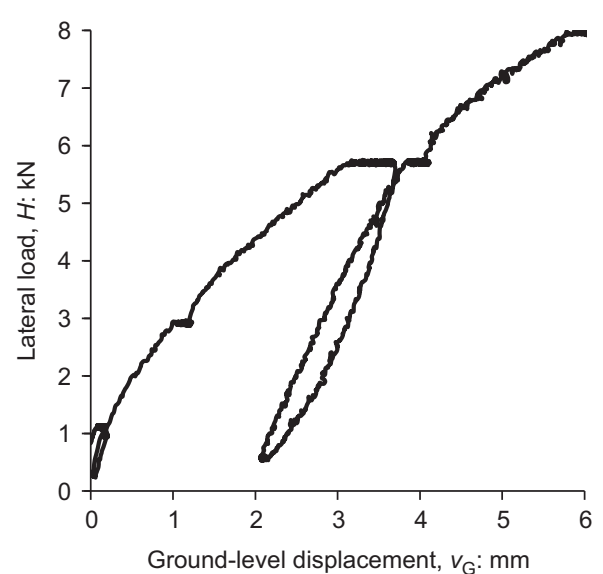

(a)

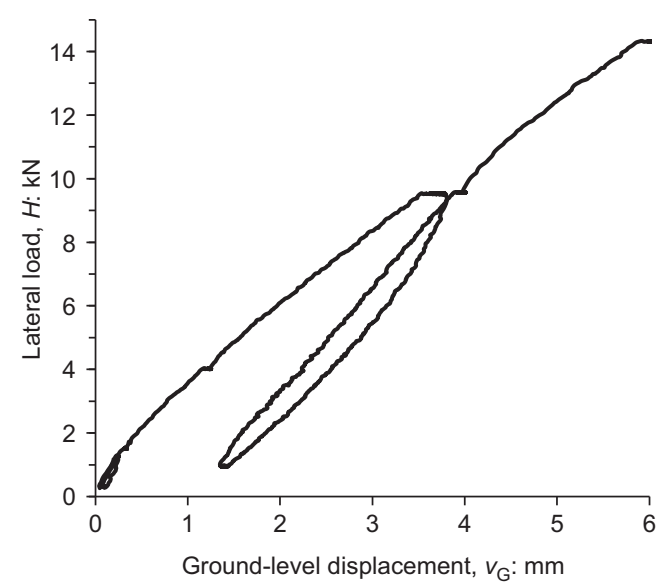

(c)

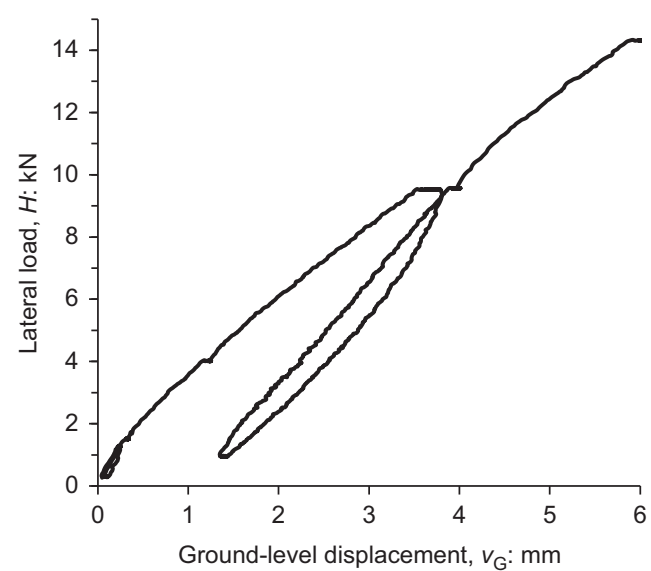

(b)

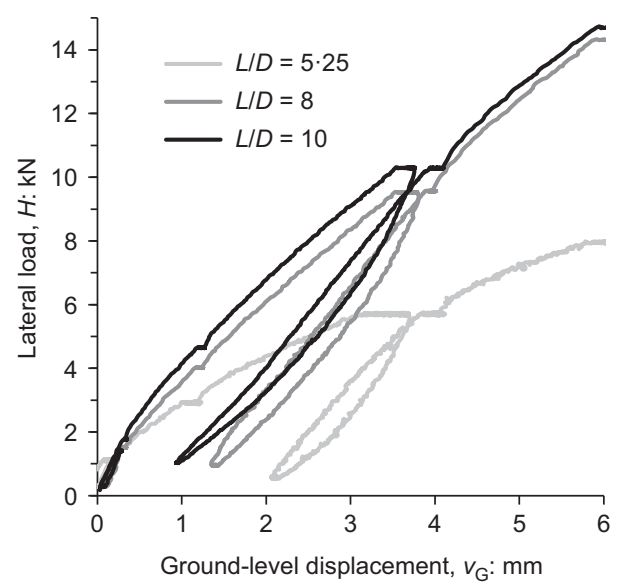

(d)

Fig. 5. Comparison of load-displacement response for $D=0 \cdot 273 \mathrm{~m}$ diameter piles with different length-to-diameter ratios during initial load stages 1-4: (a) $L I D=5 \cdot 25$ (DS1); (b) $L I D=8$ (DS3); (c) $L I D=10$ (DS4); (d) three $L I D$ ratios

MEDIUM-DIAMETER $(D=0.762 \mathrm{~m})$ TEST RESULTS

The majority of tests were conducted with a pile diameter of $0.762 \mathrm{~m}$, and explored the influences of pile length and loading rate.

\section{Repeatability}

Two similar tests provided a check on repeatability, DM5 and DM7 $(L / D=3)$, as shown in Fig. 6(a). Pile DM5 was the first of the two piles to be tested. During the creep period of load stage 6 , the load capacity of the pile was found to reduce, resulting in an acceleration of the loading system (which was set to maintain the target load), followed by a brief period of oscillation and measurement noise. During the repeated test, DM7, in anticipation that similar softening behaviour might occur, the control system was set to control displacement, at a rate of $D / 300 \mathrm{~min}^{-1}$, immediately after the displacement rate during the constant load creep period reached $D / 300 \mathrm{~min}^{-1}$ (i.e. the displacement rate used for the main monotonic loading stages). Owing to concerns that the test pile may become unstable, it was decided to conclude the DM7 test before the planned ultimate ground-level displacement $(0 \cdot 1 D)$ had been reached. Fig. 6(a) indicates that the two sets of test results are in good agreement; this provides confidence of the repeatability of the results obtained from the test programme.

The initial load stages for the three medium-length $(L / D=5 \cdot 25)$ pile tests are shown in Fig. 6(b). Pile DM9 was used as the reaction pile for the small-diameter pile tests and was subsequently loaded in the medium-diameter pile test series. Piles DM9 and DM4 were both loaded using the standard monotonic load procedure (Table 2). The data in Fig. 6(c) indicate good agreement between these tests, providing further confirmation of the repeatability of the results. Following successful tests on piles DM4 and DM9, it was decided to vary the loading regime for pile DM6 after the first four monotonic load stages to explore the effect of loading rate through two alternate displacement rates (described in more detail later). For this reason, data for DM6 are included in Fig. 6(b) only for the first four monotonic load stages. It is clear that the data for DM4 and DM6 match each other very closely. Pile DM9 exhibits a slightly softer response; it is possible that the performance of this pile had been influenced by its previous use as a reaction pile.

Response of piles with different length-to-diameter ratios

The ground-level load-displacement response for the three medium-diameter pile tests with $L / D=3,5 \cdot 25$ and 8 is shown in Fig. 7. The three responses are compared in Fig. 7(d). As expected, longer piles exhibited a greater capacity and tangential stiffness at the stage when the test was terminated, at $v_{\mathrm{G}}=D / 10$ and $\theta_{\mathrm{G}}=2^{\circ}$. Residual displacements are seen to develop when the load is removed, but the magnitudes of the residual displacements do not vary in a systematic way with changes in embedded pile length, due to differing maximum displacements prior to unloading. 


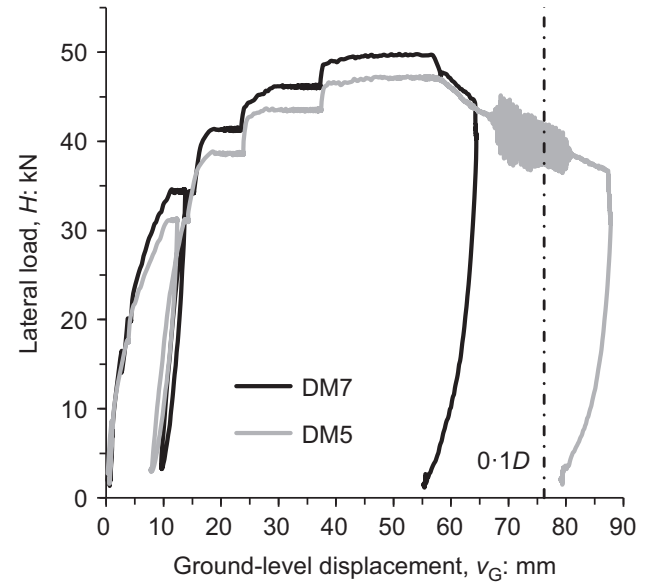

(a)

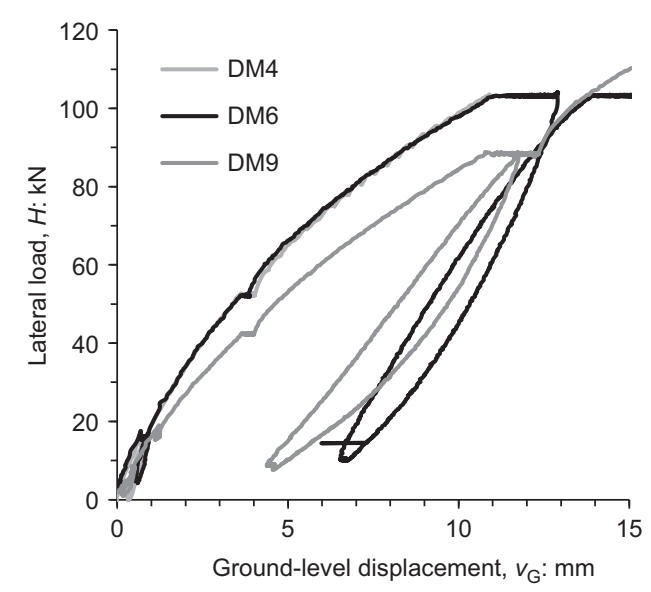

(b)

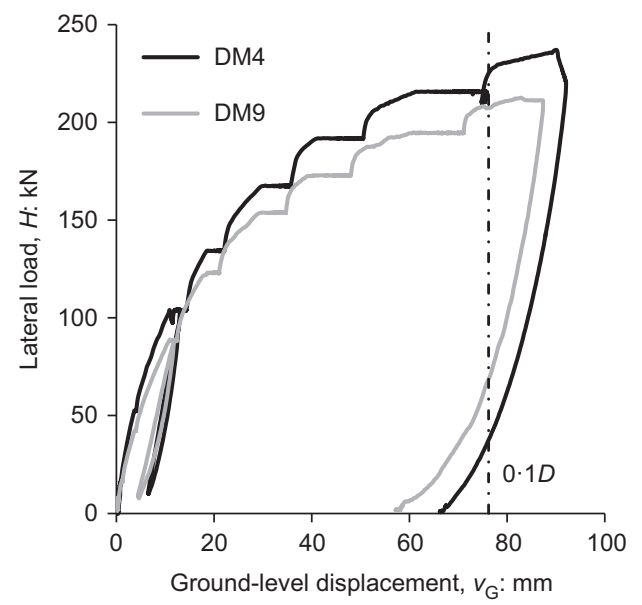

(c)

Fig. 6. Repeat medium-diameter $(D=0 \cdot 762 \mathrm{~m})$ pile tests: (a) $L I D=3$; (b) $L I D=5 \cdot 25$; (c) $L I D=5 \cdot 25$

The interpreted ground-level load-displacement response during the initial load stages is shown in Fig. 8. The response of DM7 $(L / D=3)$, shown in Fig. 8(a), indicates some small unload-reload loops during load stage 2; this unplanned feature of the loading path occurred as a consequence of a marginal imbalance in the proportional and integral gain components of the control feedback loop. The gains were adjusted for the subsequent loading stages. These small unload-reload loops are not expected to influence the observed backbone response.

Figure 8(c) shows an unusual response during the unloadreload loops for the longer DM3 pile $(L / D=8)$, in which the tangential stiffness is seen to increase slightly during the reload phase. It is thought that this behaviour is associated with the presence of gapping around the pile. When the pile is unloaded, a gap forms on the front (i.e. the passive) face of the pile. When the pile is reloaded, the gap progressively closes with the consequence that the incremental stiffness increases as the load is applied. A similar effect can be observed in Fig. 5(c) for a small-diameter pile.

Figure 8(d) indicates that increasing the length of the pile results in an increase in the stiffness of response during initial loading.

\section{LARGE-DIAMETER $(D=2 \cdot 0 \mathrm{~m})$ TEST RESULTS}

The last tests conducted at the site were on the two large-diameter $(D=2.0 \mathrm{~m})$ piles, DL1 and DL2. These piles were loaded directly against each other, resulting in two simultaneous tests. Fig. 9 shows the interpreted load-displacement responses for these piles. Similar responses were observed at both large and small displacements; this provides a further check on the repeatability of the tests. Pile DL2 achieved an interpreted ultimate load, $H_{0 \cdot 1 D}$ (where $H_{0 \cdot 1 D}$ is defined as the lateral load applied to the pile at $v_{\mathrm{G}}=0 \cdot 1 D$ ), that is $1.9 \%$ higher than that recorded for DL1.

\section{PILE RESPONSE METRICS}

Owing to the pattern of loading adopted in the tests (Table 2) the measured pile response features periods of constant velocity displacement, constant load creep and unload-reload loops. This can result in difficulty in consistently comparing the pile performance between different tests according to specific criteria, such as the load at ultimate displacement, $v_{\mathrm{G}}=D / 10$. A procedure was developed (described in the paper by Byrne et al. (2019)) to establish a cubic spline representation of the $D / 300 \mathrm{~min}^{-1}$ backbone curves in which the influence of the creep data was removed. This process was adopted here to establish the backbone curves for the tests conducted at Dunkirk, as demonstrated in Fig. 10.

Stiffness and ultimate load metrics are shown in Table 3, where $k_{\text {Hinit }}$, is the secant stiffness of the $H$ plotted against $v_{\mathrm{G}}$ response for $0 \leq v_{\mathrm{G}} \leq D / 1000$ (see Fig. $10(\mathrm{~b})$ ); $k_{\text {Minit }}$ is the secant stiffness of the $M_{\mathrm{G}}$ plotted against $\theta_{\mathrm{G}}$ response for $0 \leq \theta_{\mathrm{G}} \leq(2 / 100)^{\circ}$. The reload stiffness, $k_{\mathrm{Hreload}}$, is the secant slope of the unload-reload loop in the $H$ plotted against $v_{\mathrm{G}}$ response following load stage 3 ; this is determined from the incremental response for loading displacement of $\Delta v_{\mathrm{G}}=D / 1000$ immediately following the resumption of 


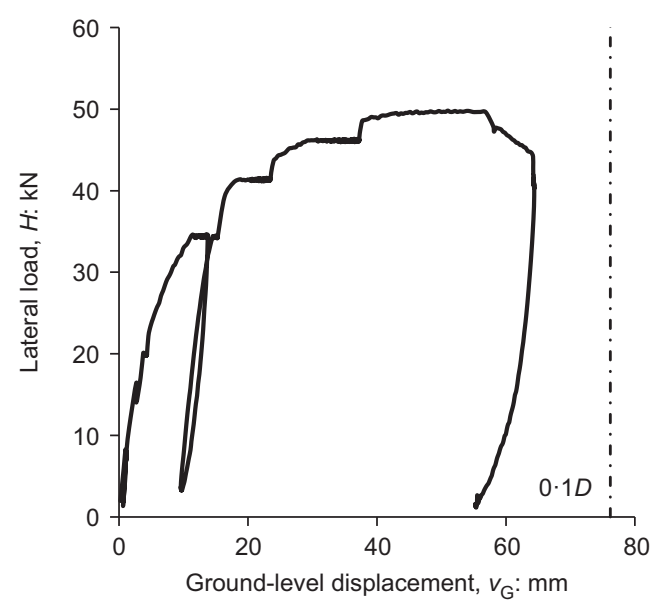

(a)

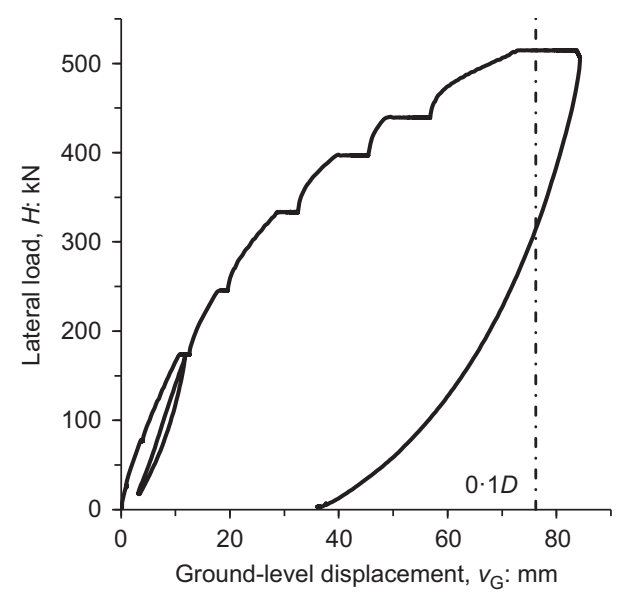

(c)

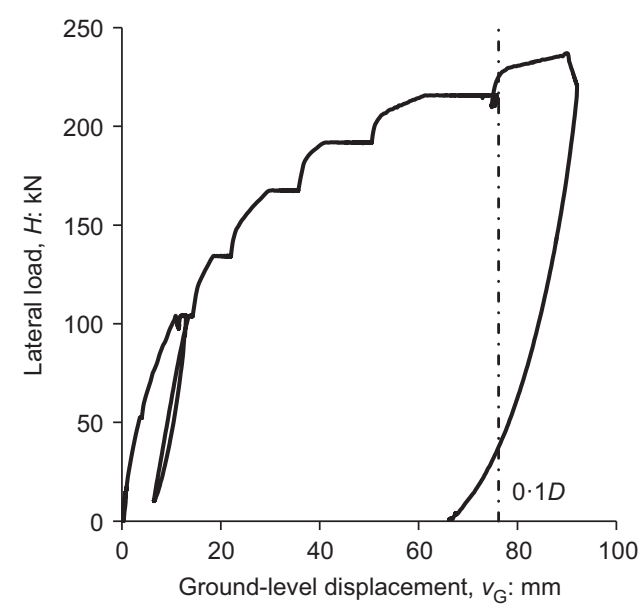

(b)

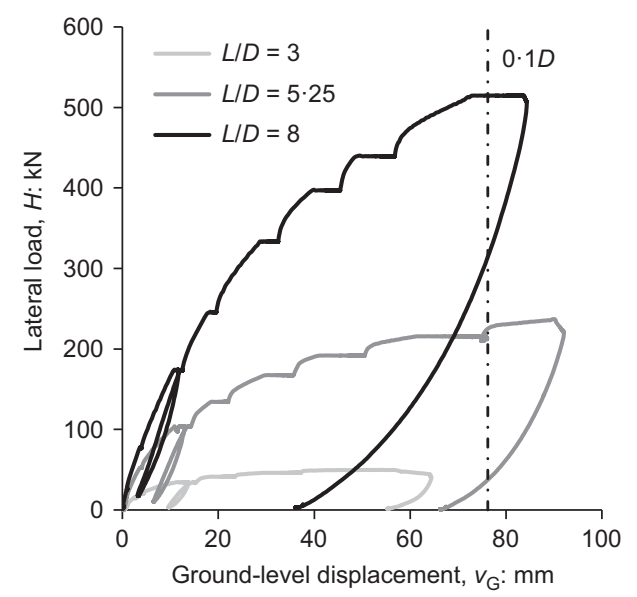

(d)

Fig. 7. Comparison of load-displacement response for $D=0 \cdot 762 \mathrm{~m}$ diameter piles with different length-to-diameter ratios: (a) $L I D=3$ (DM7); (b) $L I D=5 \cdot 25$ (DM4); (c) $L I D=8$ (DM3); (d) three $L I D$ ratios

loading following the load reversal process. The reload stiffness data were determined directly from the field results after smoothing using a Gaussian window of 21 samples' width $(2 \cdot 1 \mathrm{~s})$ in both the forward and reverse filtering directions. Also shown in Table 3 are values of $H_{0.1 D}$ and $M_{2^{\circ}}$; these data are indicative of the ultimate capacity of the pile and are determined from the cubic splines, corresponding to the values of $H$ determined at $v_{\mathrm{G}}=D / 10$ and the values of $M$ at $\theta_{\mathrm{G}}=2^{\circ}$. Note that the ultimate load indicated in Table 3 for the short $(L / D=3)$ pile is the peak capacity that was achieved. As mentioned earlier, for this particular pile the test had to be terminated early as a consequence of the development of softening behaviour.

\section{Response metric comparison}

Figure 11 indicates the variation of ultimate load, $H_{0 \cdot 1 D}$, initial stiffness, $k_{\text {Hinit }}$, and reload stiffness, $k_{\text {Hreload, with }}$ length-to-diameter ratio for the medium-diameter piles. Fig. 11 indicates that the metrics all increase with increases in pile length, as expected. However, the increase in reload stiffness with length is less significant; it is thought that this observation may be associated with gapping around the pile.

\section{RATE EFFECTS}

The complementary set of tests conducted at the Cowden site indicated that loading rate has a significant effect on the monotonic backbone response for monopiles in clay (Byrne et al., 2019). Tests were therefore included at Dunkirk that allowed the influence of loading rate to be investigated for piles in sand.

Initially, rate effects were investigated using pile DS2 $(D=0.273 \mathrm{~m}, L / D=5 \cdot 25)$. This pile was loaded at an average rate of $325 \mathrm{~mm} / \mathrm{min}$; the pile length and loading configuration is the same as pile DS1, which was loaded at an average rate of $0.91 \mathrm{~mm} / \mathrm{min}$. A comparison between the two sets of data is shown in Fig. 12(a); the test conducted at the faster rate indicates a strength enhancement of about $20 \%$ (corresponding to a $7 \cdot 5 \%$ increase per $\log _{10}$ cycle of loading rate).

A separate rate test was conducted on pile DM6 $(D=0.762 \mathrm{~m}, L / D=5.25)$ in which the initial four load stages of the standard monotonic regime were conducted, followed by alternating load stages of high (average $330 \mathrm{~mm} / \mathrm{min}$ ) and low (average $0.45 \mathrm{~mm} / \mathrm{min}$ ) displacement rate. The results, plotted in Fig. 12(b), suggest that a unique backbone curve exists at each loading rate; the inferred backbone curves are indicated as dashed lines in Fig. 12(b). These backbone curves indicate a rate-induced increase in strength of $11 \%$, corresponding to a rate effect of about $4 \%$ per $\log _{10}$ cycle. This is less significant than the influence of rate observed for the small-diameter pile tests.

\section{PILE GAPPING}

In a similar fashion to the pile tests in clay (Byrne et al., 2019), the opening of a gap was observed on the active face of all test piles (and often on the passive face after unloading). 


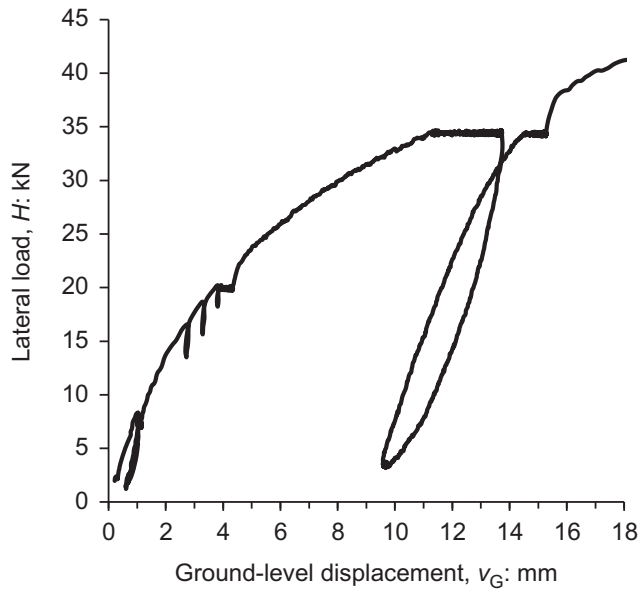

(a)

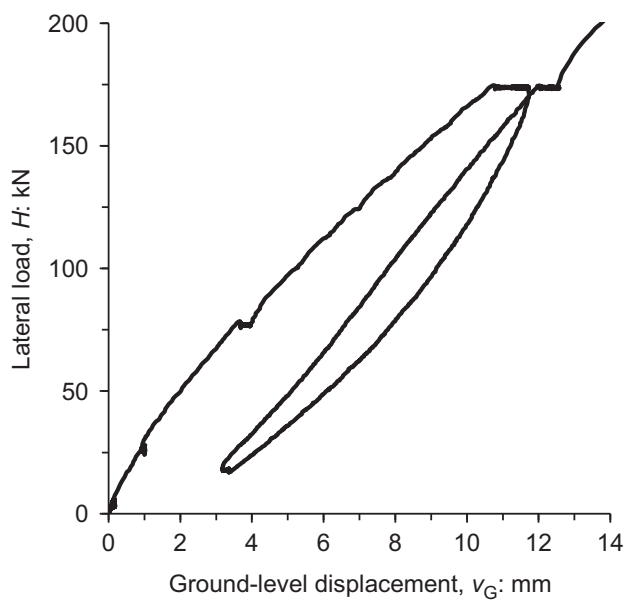

(c)

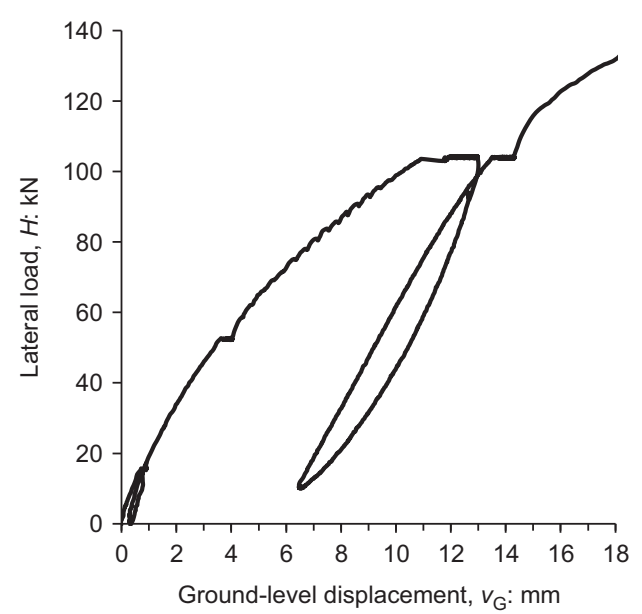

(b)

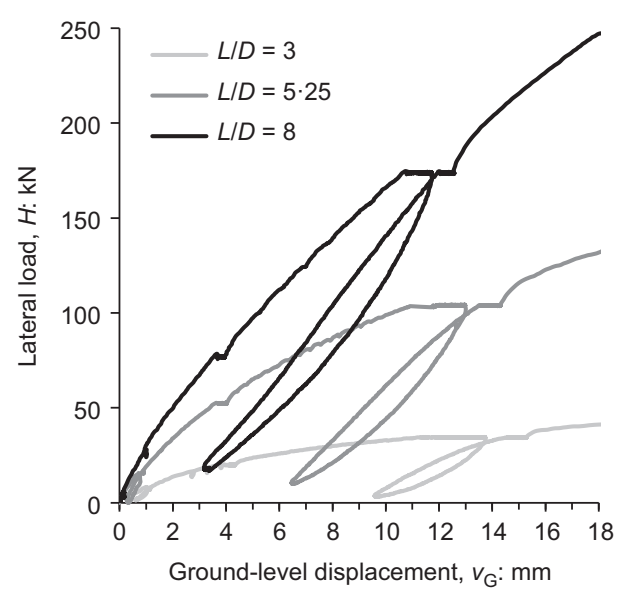

(d)

Fig. 8. Comparison of load-displacement response at small displacement for $D=0 \cdot 762$ m diameter piles with different length-to-diameter ratios: (a) $L I D=3$ (DM7); (b) $L I D=5 \cdot 25$ (DM4); (c) $L I D=8$ (DM3); (d) three $L I D$ ratios

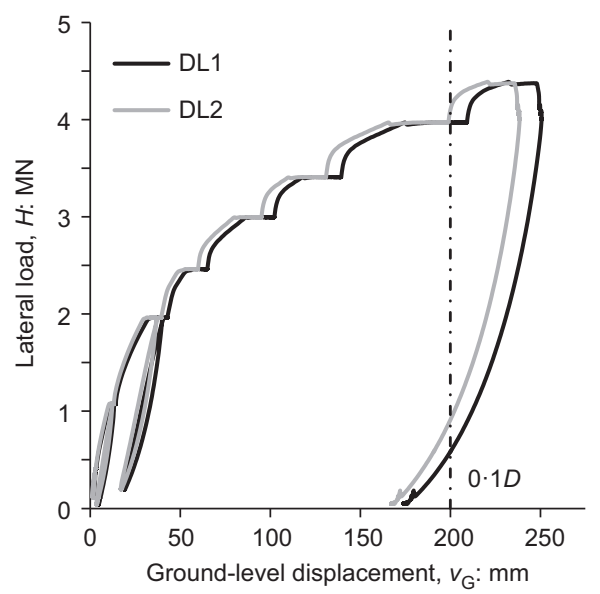

(a)

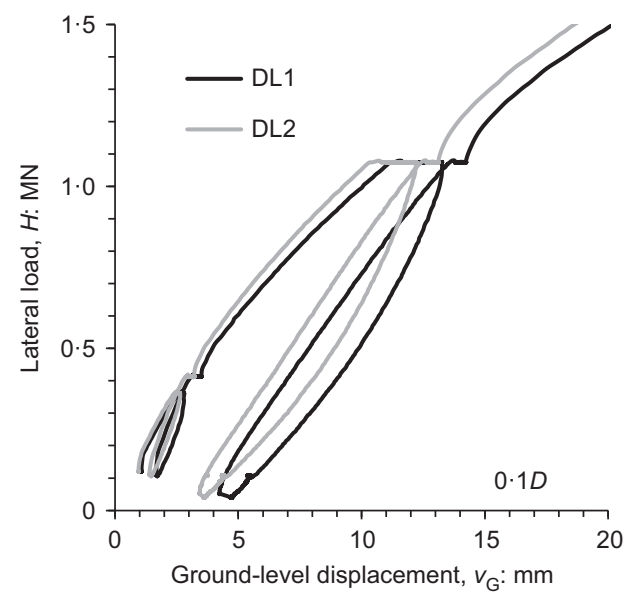

(b)

Fig. 9. Large-diameter pile response: (a) overall monotonic response; (b) small displacement response

Table 4 shows the depth of the gap below ground level on the active pile face, after the final unload stage, measured using a metal tape. The development of a gap will obviously have a significant impact on the pile response, reducing the initial stiffness and altering the shape of the loading curve, which is likely to in turn influence the overall dynamics of the pile/support structure system of full-scale installations.
It should be noted, however, that the gaps reported in Table 4 occurred as a result of loads that substantially exceed the likely in-service conditions of a foundation in the field. It should also be noted that the likely unsaturated nature of the superficial layers at the Dunkirk site means that the gap formation observed in the tests may not be indicative of offshore conditions, for which the soil is likely to be saturated. 


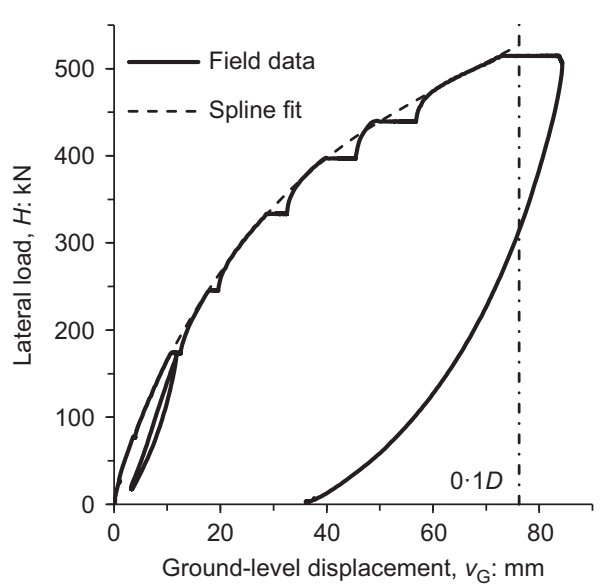

(a)

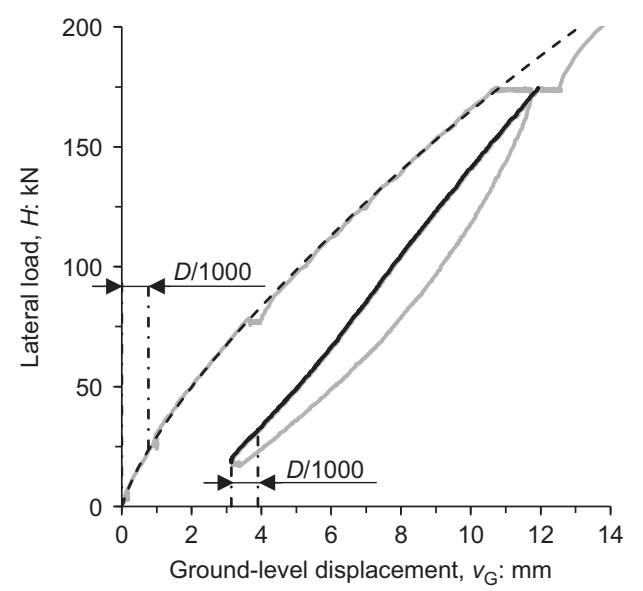

(b)

Fig. 10. Fitting of a single response curve to field test data for pile DM3 $(D=0.762 \mathrm{~m}, L / D=8)$ : (a) overall load-displacement response; (b) load stages 1-3 and unload-reload response (reloading in full black line)

Table 3. Test pile response metrics

\begin{tabular}{|c|c|c|c|c|c|c|c|}
\hline Pile & $D: \mathrm{m}$ & Nominal $L / D$ & $k_{\text {Hinit }}: \mathrm{MN} / \mathrm{m}$ & $H_{0 \cdot 1 D}: \mathrm{kN}$ & $k_{\text {Minit }}: \mathrm{kNm} / \mathrm{deg}$ & $M_{2^{\circ}}: \mathrm{kNm}$ & $k_{\text {Hreload: }}: \mathrm{MN} / \mathrm{m}$ \\
\hline DS1 & $0 \cdot 273$ & $5 \cdot 25$ & $5 \cdot 18$ & $14 \cdot 9$ & $224 \cdot 0$ & $75 \cdot 9$ & $3 \cdot 30$ \\
\hline DS2 & $0 \cdot 273$ & $5 \cdot 25$ & $4 \cdot 28$ & $17 \cdot 8$ & $208 \cdot 1$ & $92 \cdot 8$ & - \\
\hline DS3 & $0 \cdot 273$ & 8 & $4 \cdot 85$ & - & 183.4 & - & $4 \cdot 15$ \\
\hline DS4 & $0 \cdot 273$ & 10 & $5 \cdot 44$ & - & $265 \cdot 6$ & - & $2 \cdot 94$ \\
\hline DM5 & $0 \cdot 762$ & 3 & $8 \cdot 13$ & $46 \cdot 9$ & 1875 & $473 \cdot 0$ & $7 \cdot 44$ \\
\hline DM7 & $0 \cdot 762$ & 3 & $8 \cdot 07$ & $49 \cdot 6$ & 1651 & $492 \cdot 1$ & $8 \cdot 52$ \\
\hline DM4 & $0 \cdot 762$ & $5 \cdot 25$ & $22 \cdot 2$ & $226 \cdot 3$ & 4428 & 2272 & $14 \cdot 3$ \\
\hline DM9 & 0.762 & $5 \cdot 25$ & $15 \cdot 8$ & $206 \cdot 7$ & 3781 & 2064 & $10 \cdot 5$ \\
\hline DM6 & $0 \cdot 762$ & $5 \cdot 25$ & $27 \cdot 8$ & - & 4786 & - & $17 \cdot 8$ \\
\hline DM3 & $0 \cdot 762$ & 8 & $30 \cdot 8$ & $527 \cdot 1$ & 6397 & 5222 & $16 \cdot 3$ \\
\hline DL1 & $2 \cdot 0$ & $5 \cdot 25$ & $139 \cdot 7$ & 4156 & 58670 & 40010 & $91 \cdot 7$ \\
\hline DL2 & $2 \cdot 0$ & $5 \cdot 25$ & $147 \cdot 6$ & 4235 & 58160 & 40550 & $96 \cdot 3$ \\
\hline
\end{tabular}

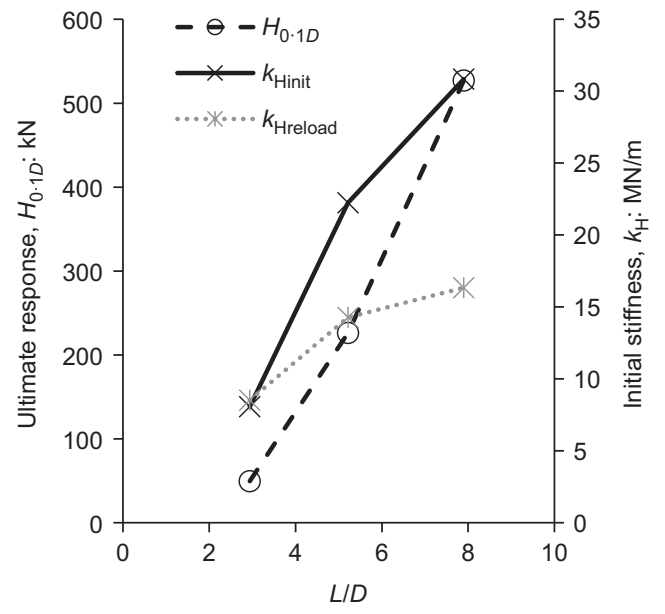

Fig. 11. Comparison of pile response metrics with length-to-diameter ratio for a pile diameter of $D=0.762 \mathrm{~m}$

\section{INFERENCES ON THE EMBEDDED PILE RESPONSE}

The performance of the embedded length of the piles has been inferred from the below-ground fibre optic strain gauges and inclinometers using the structural optimisation approach described in the paper by Byrne et al. (2019). In this approach, the embedded pile is represented as a series of Timoshenko beam elements. An 'equivalent' distributed lateral load, $p$, is assumed to act on the pile; a piecewise linear function is adopted to represent the depth variation of the equivalent distributed load. The structural model is optimised to provide a best fit with the data on bending moment and inclination implied by the below-ground instruments. The optimisation process also ensures that the bending moment, shear force, lateral pile displacement and pile rotation implied by the model at the ground surface are consistent with information provided by the above-ground instruments. In the application of this optimisation approach to the Cowden test data (Byrne et al., 2019), a limiting condition was placed on the shear force at the pile toe. For the Dunkirk tests, however, no limiting conditions were applied at the pile toe.

This optimisation approach exploits the available redundancy in the measurements; as a consequence, the reliability of inferences made on the below-ground displacements and bending moments is improved. Example results obtained from this process are described below.

A comparison between the measured data and the corresponding optimised structural model for DM3 $(D=0.762 \mathrm{~m}$, $L / D=8$ ) for $v_{\mathrm{G}}=49 \mathrm{~mm}$ (corresponding to $0 \cdot 06 \mathrm{D}$ ) is shown in Fig. 13. The structural model is seen to provide a good fit with the measurements. Also shown (Fig. 13(e)) is the inferred distribution of the equivalent distributed lateral load. Fig. 14 shows plots of lateral deflection and bending moment for DM3, determined at additional stages of the test, where $z$ is the distance beneath the ground surface. At small loads, the pile exhibits a flexural mode of deflection, where the pile toe remains relatively 


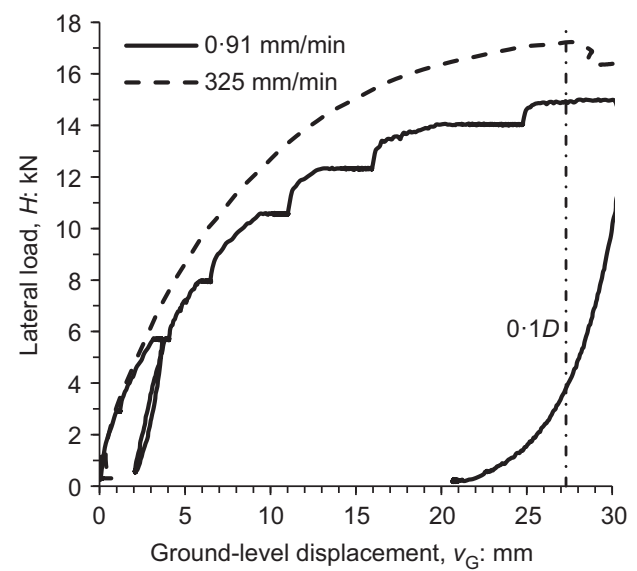

(a)

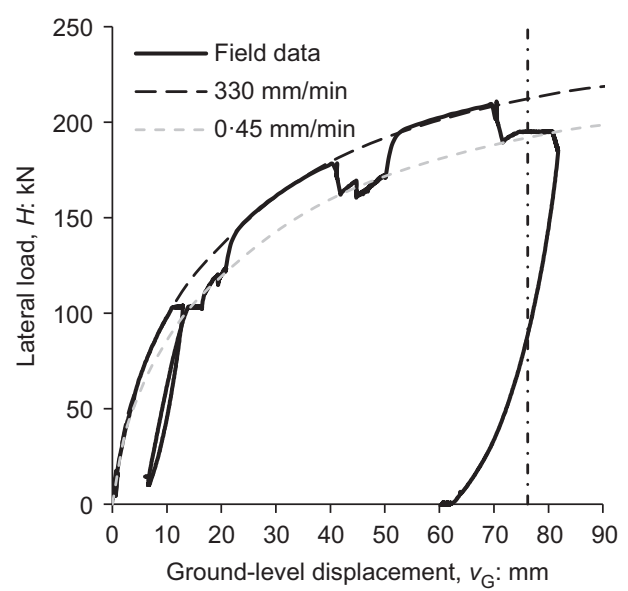

(b)

Fig. 12. Varied loading rate tests: (a) DS1 and DS2 $(D=0 \cdot 273 \mathrm{~m}, L I D=5 \cdot 25)$; (b) DM6 $(D=0 \cdot 762 \mathrm{~m}, L I D=5 \cdot 25)$

Table 4. Test pile active face gap depths

\begin{tabular}{|c|c|c|c|}
\hline Pile & Installed $L:(\mathrm{m})$ & Gap depth, $L_{\mathrm{G}}: \mathrm{m}$ & $L_{\mathrm{G}} / L$ \\
\hline DS1 & $1 \cdot 43$ & 0.68 & $0 \cdot 48$ \\
\hline DS2 & $1 \cdot 43$ & 0.535 & $0 \cdot 37$ \\
\hline DS3 & $2 \cdot 18$ & - & - \\
\hline DS4 & $2 \cdot 73$ & 0.79 & $0 \cdot 29$ \\
\hline DM5 & $2 \cdot 27$ & $1 \cdot 17$ & 0.52 \\
\hline DM7 & $2 \cdot 24$ & $1 \cdot 15$ & 0.51 \\
\hline DM4 & 3.98 & - & - \\
\hline DM9 & 3.96 & 1.67 & $0 \cdot 42$ \\
\hline DM6 & 3.99 & $1 \cdot 84$ & $0 \cdot 46$ \\
\hline DM3 & $6 \cdot 02$ & 2.98 & $0 \cdot 50$ \\
\hline DL1 & $10 \cdot 61$ & $3 \cdot 56$ & $0 \cdot 34$ \\
\hline DL2 & $10 \cdot 57$ & $4 \cdot 25$ & $0 \cdot 40$ \\
\hline
\end{tabular}

In all cases a gap was seen to form between the pile and the soil at the end of the test. The absence of data for two of the piles indicates that the gap depths were not recorded for these piles.

fixed. The depth of the pivot point (i.e. the point at which the lateral displacement is zero) progressively increases as the load is increased, until reaching an asymptotic value of approximately $z / D=0 \cdot 61$. During the transition of the pivot point a toe kick develops and the pile exhibits a combined flexural and rotational deflected shape. Fig. 14(b) shows that as the load is increased, the depth of the peak bending moment also increases; which is likely to be due to the transfer of the applied load to soil reactions at greater depths.

Inferred data on embedded pile displacements and bending moments for DM7 $(D=0.762 \mathrm{~m}, L / D=3)$ are shown in Fig. 15. In this case, the pile exhibits a rigid rotational deflection at all applied loads. The normalised pivot depth of approximately $z / D=0.74$ appears to remain unchanged throughout the test. As shown in Fig. 15(b), the depth of the peak bending moment remains relatively constant and shallow throughout the loading process. Also shown on Fig. 15 are bending moments implied by the fibre optic strain gauges. In this case, a significant spread is observed in the bending moments at each measurement depth. This large spread of data is unusual (the pattern in Fig 13(b) is more typical) and the detailed causes of the spread are uncertain in this case. However, it is considered that the bending moment distributions implied by the optimised model are reasonably robust in this case, since they are partly determined by data from other instruments. The optimised model implies a significant pile toe moment $(70 \mathrm{kNm}$ at $H=46 \mathrm{kN})$. The stress conditions at the base of the pile are difficult to estimate and so assessing the extent to which this bending moment is realistic is not straightforward. It is noted, however, that a significant pile toe bending moment is also observed in the Cowden tests (Byrne et al., 2019) for pile CM2 (which is equivalent to the DM7 pile being considered here).

Figure 16 shows data inferred from one of the largediameter piles (DL2, $D=2 \cdot 0 \mathrm{~m}, L / D=5 \cdot 25$ ), which indicates that the pile is sufficiently slender to exhibit an initially flexible deflected shape at small loads. As the test progresses and the load is increased, the pile deflection transitions to a relatively rigid rotational mode with toe kick, and a final normalised pivot depth of approximately $z / D=0 \cdot 68$. In a similar fashion to DM3, the depth of the peak moment is observed to increase as the load is increased. Bending moments inferred from the fibre optic strain gauges are seen to exhibit a significant spread in the lower half of the pile.

\section{CONCLUSIONS}

The conclusions from the current study are broadly consistent with many of the findings of the related Cowden study (Byrne et al., 2019). The following conclusions, in particular, can be noted.

(a) Data are presented from monotonic laterally loaded pile tests, conducted at Dunkirk, principally in terms of load-deflection response for piles of three different diameters and a range of length-to-diameter ratios.

(b) A high level of repeatability was observed from tests in which the same pile configurations were employed. This provides confidence in the test processes and the data interpretation procedures. This finding supports the use of the data for verifying the $3 \mathrm{D}$ finite-element models that have been developed to aid the development of the PISA modelling framework.

(c) Softening behaviour was observed in the short, medium-diameter piles $(D=0.762 \mathrm{~m}, L / D=3)$. It is noted that softening behaviour was not observed for any of the complementary tests conducted at the Cowden clay site.

(d) For the medium-diameter piles, strength and stiffness metrics were all found to increase with increasing length, as expected. 


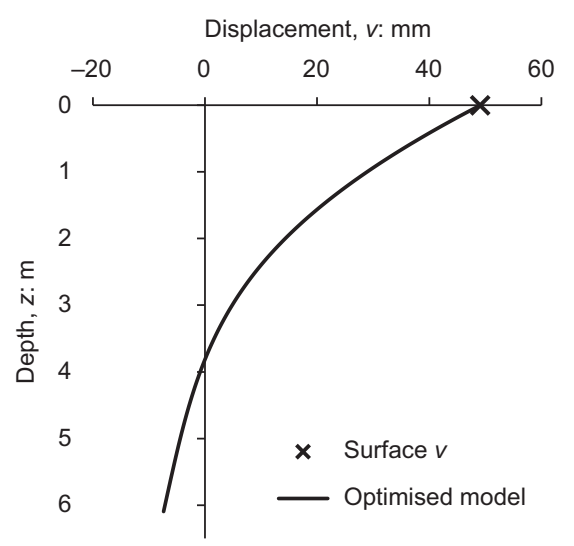

(a)

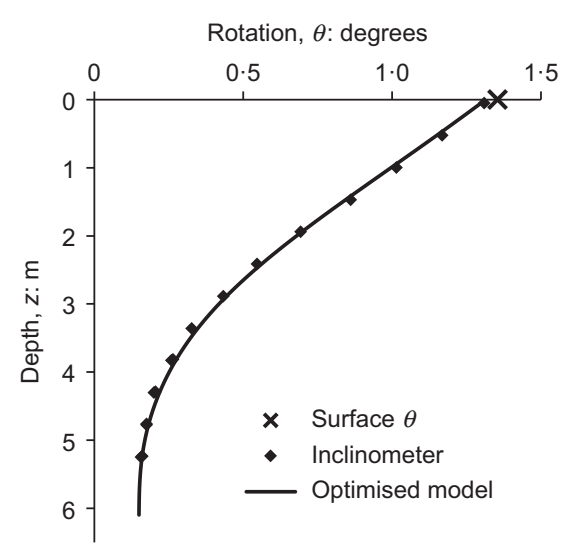

(b)

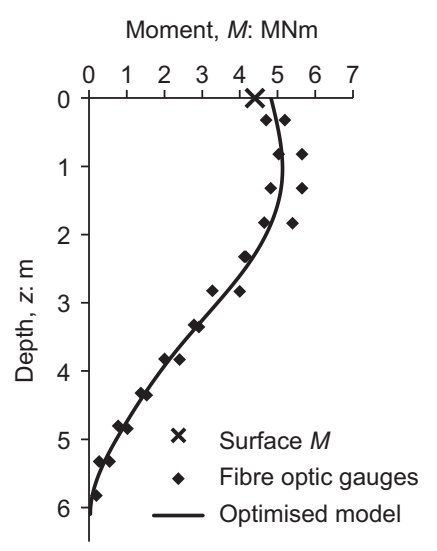

(c)

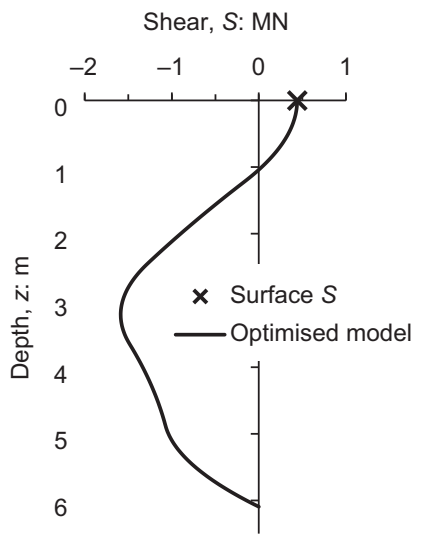

(d)

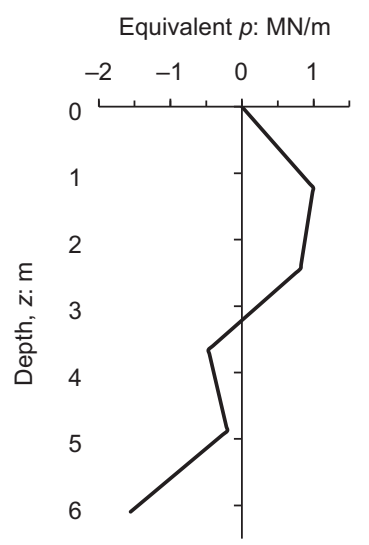

(e)

Fig. 13. Embedded pile response for pile DM3 $(D=0.762 \mathrm{~m}, L I D=8)$ at $H=440 \mathrm{kN}, v_{\mathrm{G}}=49 \mathrm{~mm}$. Parts (a) and (b) indicate the inferred data on ground-level displacement and rotation (indicated as 'Surface $v$ ' and 'Surface $\theta$ ', respectively) determined from the above-ground instrumentation, using the methods described in the paper by Burd et al. (2019). Parts (c) and (d) indicate ground-level bending moment and shear force (indicated as 'Surface $M$ ' and 'Surface $S$ ', respectively) determined directly from the applied load. Part (e) indicates the equivalent distributed lateral load determined from the optimised model

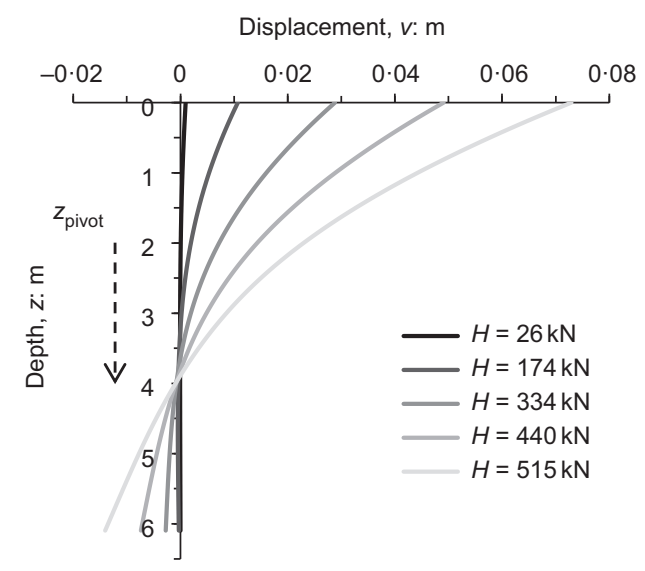

(a)

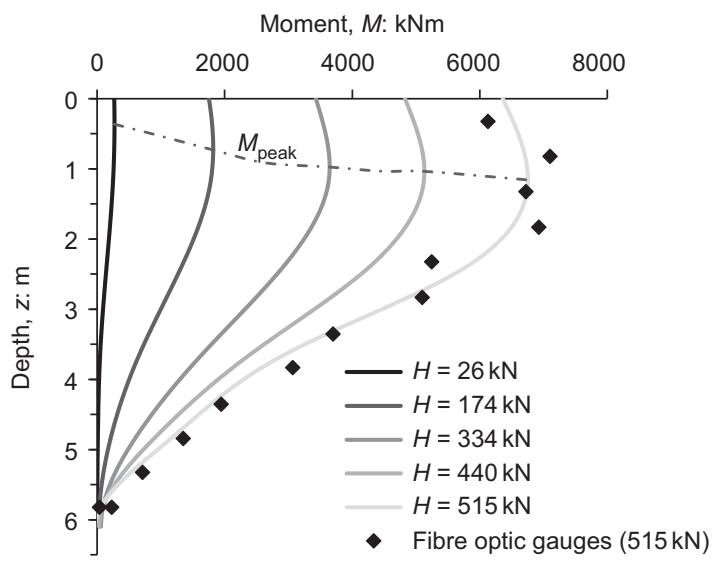

(b)

Fig. 14. Profiles of (a) displacement and (b) bending moment for pile DM3, including bending moment data inferred directly from the fibre optic strain gauges at $H=515 \mathrm{kN}$

(e) Tests conducted at different velocities on both small- and medium-diameter piles indicated an enhancement in strength at higher rates of loading. Although the magnitude of this rate effect cannot be precisely quantified from the current data, it seems plausible that monopiles in sand have reserves of strength at high loading rates that are not considered in current design approaches. Note, however, that the influence of loading rate is less strong in sand at Dunkirk than in the complementary tests at the clay site in Cowden (Byrne et al., 2019).

( $f$ ) Time-dependent effects - assumed to be due to creep are apparent in all of the data from the monotonic loading tests. 


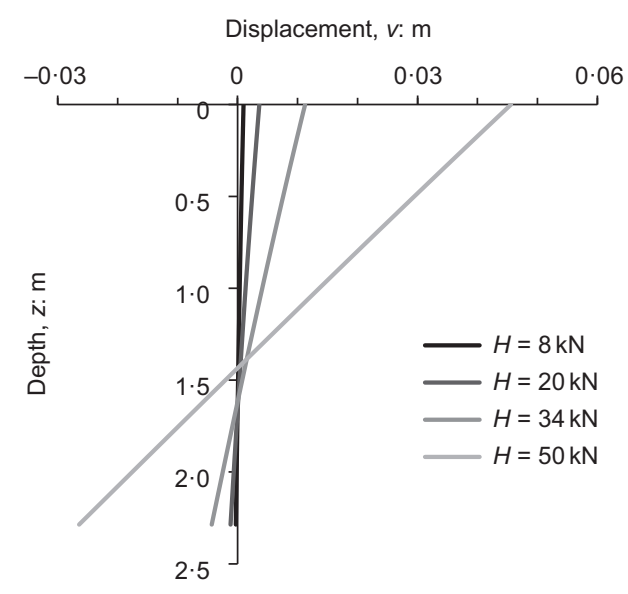

(a)

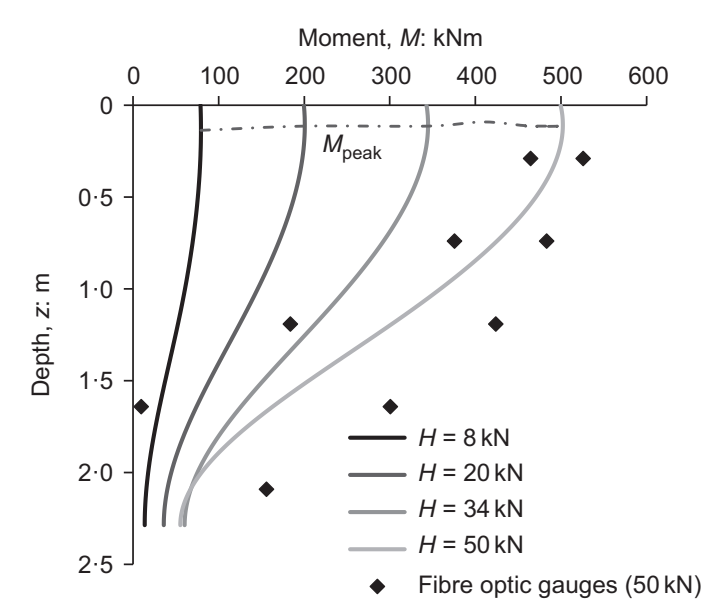

(b)

Fig. 15. Profiles of (a) displacement and (b) bending moment for pile DM7, including bending moment data inferred directly from the fibre optic strain gauges at $H=50 \mathrm{kN}$

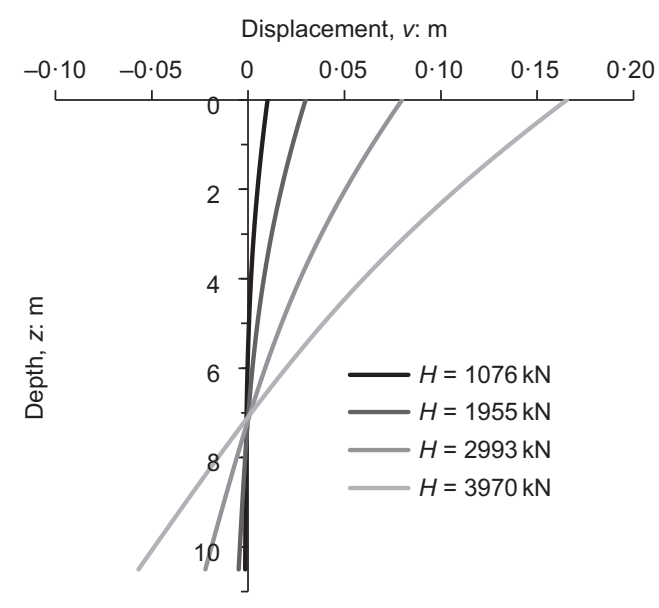

(a)

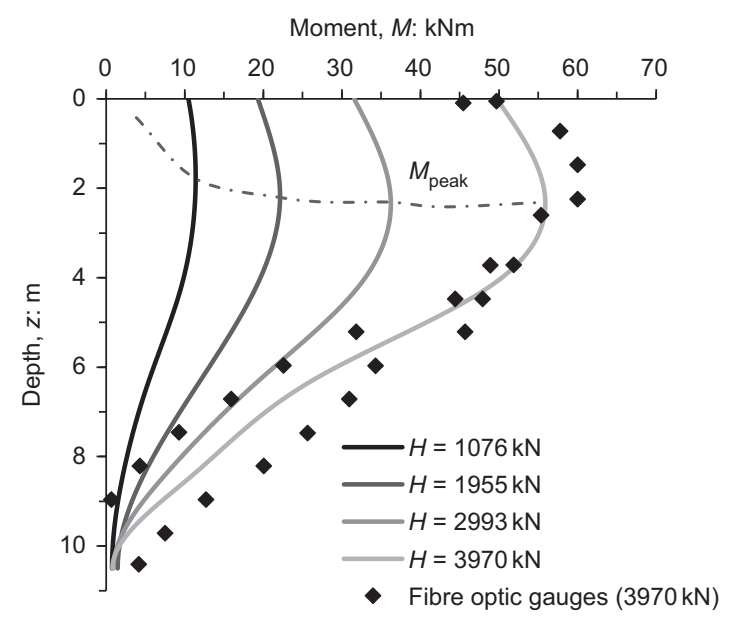

(b)

Fig. 16. Profiles of (a) displacement and (b) bending moment for pile DL2, including bending moment data inferred directly from the fibre optic strain gauges at $H=3970 \mathrm{kN}$

(g) The assumed pore pressure suctions associated with the superficial layer are likely to have permitted the development of gaps around the pile. These gaps will have influenced the observed unload-reload behaviour. The suctions are unlikely to be representative of offshore conditions. Ideally, the tests would have been conducted offshore, but this would have considerably increased the costs and complexity of the project.

(h) The data form the empirical database to be used for validation of finite-element analyses, which in turn is used to develop a new one-dimensional modelling procedure for offshore monopile design (Byrne et al., 2017; Taborda et al., 2019; Zdravković et al., 2019).

\section{ACKNOWLEDGEMENTS}

The PISA project was funded by the UK Department for Energy and Climate Change (DECC) and the PISA industry partners under the umbrella of the offshore wind accelerator (OWA) programme which was designed and is led by the Carbon Trust. The authors acknowledge the provision of financial and technical support by the following project partners: Ørsted Wind Power (formerly DONG Energy), Alstom Wind, E.ON, EDF, Equinor (formerly Statoil), innogy, SPR, SSE, Vattenfall and Van Oord. The authors acknowledge gratefully the work of Socotec UK Ltd (formerly ESG) as the main contractor for the design and execution of the field testing programme.

\section{NOTATION}

$B$ small strain shear modulus parameter

$D$ pile diameter

$e$ voids ratio

$G_{0} \quad$ small strain shear modulus

$H$ applied lateral load

$h$ height of point of application of lateral load above ground level

$k_{\mathrm{H}} \quad$ secant stiffness $\Delta H / \Delta v_{\mathrm{G}}$

$k_{\mathrm{M}} \quad$ secant stiffness $\Delta M_{\mathrm{G}} / \Delta \theta_{\mathrm{G}}$

$L$ pile embedded length

$L_{\mathrm{G}} \quad$ measured depth of gap on active face of pile

$M$ bending moment in pile

$M_{\mathrm{G}}$ moment at ground level, $M_{\mathrm{G}}=H h$

$p^{\prime} \quad$ mean effective stress 
$t$ pile wall thickness

$v$ horizontal displacement of pile

$v_{\mathrm{G}}$ horizontal displacement of pile at ground level

$z$ depth below ground surface

$\theta_{\mathrm{G}}$ rotation of pile neutral axis at ground level

$\phi_{\mathrm{cs}}^{\prime} \quad$ critical state friction angle

\section{REFERENCES}

Aghakouchak, A. (2015). Advanced laboratory studies to explore the axial cyclic behaviour of driven piles. $\mathrm{PhD}$ thesis, Imperial College London, London, UK.

Burd, H. J., Beuckelaers, W. J. A. P., Byrne, B. W., Gavin, K. G., Houlsby, G. T., Igoe, D. J. P., Jardine, R. J., Martin, C. M., McAdam, R. A., Muir Wood, A., Potts, D. M., Skov Gretlund, J., Taborda, D. M. G. \& Zdravković, L. (2019). New data analysis methods for instrumented mediumscale monopile field tests. Géotechnique, https://doi.org/10.1680/ jgeot.18.PISA.002.

Byrne, B. W., McAdam, R. A., Burd, H. J., Houlsby, G. T., Martin, C. M., Beuckelaers, W. J. A. P., Zdravković, L., Taborda, D. M. G., Potts, D. M., Jardine, R. J., Ushev, E., Liu, T., Abadias, D., Gavin, K., Igoe, D., Doherty, P., Skov Gretlund, J., Pacheco Andrade, M., Muir Wood, A., Schroeder, F. C., Turner, S. \& Plummer, M. A. L. (2017). PISA: new design methods for offshore wind turbine monopiles. In Proceedings of the 8th international conference on offshore site investigation and geotechnics, smarter solutions for offshore developments, vol. 1, pp. 142-161. London, UK: Society for Underwater Technology.

Byrne, B. W., McAdam, R. A., Burd, H. J., Beuckelaers, W. J. A. P., Gavin, K. G., Houlsby, G. T., Igoe, D. J. P., Jardine, R. J., Martin, C. M., Muir Wood, A., Potts, D. M., Skov Gretlund, J.,
Taborda, D. M. G. \& Zdravković, L. (2019). Monotonic laterally loaded pile testing in a stiff glacial clay till at Cowden. Géotechnique, https://doi.org/10.1680/jgeot.18. PISA.003.

Chow, F. (1997). Investigations into the behaviour of displacement piles for offshore foundations. $\mathrm{PhD}$ thesis, Imperial College, University of London, London, UK.

Hardin, B. O. (1978). The nature of stress-strain behaviour for soils. In Earthquake engineering and soil dynamics - proceedings of the ASCE Geotechnical Engineering Division specialty conference, June 19-21, Pasadena, CA, USA, vol. 1, pp. 3-90. New York, NY, USA: American Society of Civil Engineers.

Taborda, D. M. G., Zdravković, L., Potts, D. M., Burd, H. J., Byrne, B. W., Gavin, K. G., Houlsby, G. T., Jardine, R. J., Liu, T., Martin, C. M. \& McAdam, R. A. (2019). Finite-element modelling of laterally loaded piles in a dense marine sand at Dunkirk. Géotechnique, https://doi.org/10.1680/jgeot.18.PISA. 006.

Zdravković, L., Taborda, D. M. G., Potts, D. M., Jardine, R. J., Sideri, M., Schroeder, F. C., Byrne, B. W., McAdam, R. A., Burd, H. J., Houlsby, G. T., Martin, C. M., Gavin, K., Doherty, P., Igoe, D., Muir Wood, A., Kallehave, D. \& Skov Gretlund, J. (2015). Numerical modelling of large diameter piles under lateral loading. In Frontiers in offshore geotechnics III (ed. V. Meyer), pp. 759-764. Leiden, the Netherlands: CRC Press/Balkema.

Zdravković, L., Jardine, R. J., Taborda, D. M. G., Abadias, D., Burd, H. J., Byrne, B. W., Gavin, K. G., Houlsby, G. T., Igoe, D. J. P., Liu, T., Martin, C. M., McAdam, R. A., Muir Wood, A., Potts, D. M., Skov Gretlund, J. \& Ushev, E. (2019). Ground characterisation for PISA pile testing and analysis. Géotechnique, https://doi.org/10.1680/jgeot.18. PISA.001. 